\title{
Phase-Amplitude Coupling and Phase Synchronization Between Medial Temporal, Frontal and Posterior Brain Regions Support Episodic Autobiographical Memory Recall
}

\author{
Nicolas Roehri ${ }^{1} \cdot$ Lucie Bréchet $^{2,3,4} \cdot$ Martin Seeber $^{1} \cdot$ Alvaro Pascual-Leone $^{3,4,5} \cdot$ Christoph M. Michel $^{1,2}$ (D)
}

Received: 7 September 2021 / Accepted: 13 January 2022 / Published online: 26 January 2022

(c) The Author(s) 2022

\begin{abstract}
Episodic autobiographical memory (EAM) is a complex cognitive function that emerges from the coordination of specific and distant brain regions. Specific brain rhythms, namely theta and gamma oscillations and their synchronization, are thought of as putative mechanisms enabling EAM. Yet, the mechanisms of inter-regional interaction in the EAM network remain unclear in humans at the whole brain level. To investigate this, we analyzed EEG recordings of participants instructed to retrieve autobiographical episodes. EEG recordings were projected in the source space, and time-courses of atlas-based brain regions-of-interest (ROIs) were derived. Directed phase synchrony in high theta $(7-10 \mathrm{~Hz})$ and gamma $(30-80 \mathrm{~Hz})$ bands and high theta-gamma phase-amplitude coupling were computed between each pair of ROIs. Using network-based statistics, a graph-theory method, we found statistically significant networks for each investigated mechanism. In the gamma band, two sub-networks were found, one between the posterior cingulate cortex (PCC) and the medial temporal lobe (MTL) and another within the medial frontal areas. In the high theta band, we found a PCC to ventromedial prefrontal cortex (vmPFC) network. In phase-amplitude coupling, we found the high theta phase of the left MTL biasing the gamma amplitude of posterior regions and the vmPFC. Other regions of the temporal lobe and the insula were also phase biasing the vmPFC. These findings suggest that EAM, rather than emerging from a single mechanism at a single frequency, involves precise spatiotemporal signatures mapping on distinct memory processes. We propose that the MTL orchestrates activity in vmPFC and PCC via precise phase-amplitude coupling, with vmPFC and PCC interaction via high theta phase synchrony and gamma synchronization contributing to bind information within the PCC-MTL sub-network or valuate the candidate memory within the medial frontal sub-network.
\end{abstract}

Keywords Episodic autobiographical memory $\cdot$ Phase synchronization $\cdot$ Phase-amplitude coupling $\cdot$ EEG $\cdot$ Network

\begin{tabular}{|c|c|}
\hline \multicolumn{2}{|c|}{ Abbreviations } \\
\hline EAM & Episodic autobiographical memory \\
\hline PAC & Phase-amplitude coupling \\
\hline ROI & Region of interest \\
\hline
\end{tabular}

Handling Editor: Micah M. Murray.

Nicolas Roehri and Lucie Bréchet have contributed equally to this work.

Christoph M. Michel

christoph.michel@unige.ch

1 Functional Brain Mapping Laboratory, Department of Fundamental Neurosciences, Campus Biotech, University of Geneva, 9 chemin des Mines, 1211 Geneva, Switzerland

2 Center for Biomedical Imaging (CIBM), Lausanne and Geneva, 1015 Lausanne, Switzerland
dPLI Directed Phase Lag Index

nMI Normalized Modulation Index

NBS Network-based statistic

dFPN Dorsal Frontoparietal Network

MTL Medial temporal lobe

ACC Anterior cingulate cortex

MCC Median cingulate cortex

PCC Posterior cingulate cortex

3 Hinda and Arthur Marcus Institute for Aging Research and Deanna and Sidney Wolk Center for Memory Health, Hebrew SeniorLife, Boston, MA, USA

4 Department of Neurology, Harvard Medical School, Boston, MA, USA

5 Guttmann Brain Health Institute, Institut Guttman de Neurorehabilitació, Barcelona, Spain 


$\begin{array}{ll}\text { SFG } & \text { Superior frontal gyrus } \\ \text { SFGmedOrb } & \text { Medial orbital part of the SFG } \\ \text { pSFG } & \text { Posterior superior frontal gyrus } \\ \text { vmPFC } & \text { Ventromedial prefrontal cortex } \\ \text { SCG } & \text { Subcallosal gyrus } \\ \text { Rec } & \text { Gyrus rectus } \\ \text { STG } & \text { Superior temporal gyrus } \\ \text { MTG } & \text { Middle temporal gyrus } \\ \text { ITG } & \text { Inferior temporal gyrus } \\ \text { PrCu } & \text { Precuneus, } \\ \text { SPG } & \text { Superior parietal gyrus } \\ \text { IPG } & \text { Inferior parietal gyrus } \\ \text { SMG } & \text { Supramarginal gyrus } \\ \text { PHG } & \text { Parahippocampal gyrus } \\ \text { HIPP } & \text { Hippocampus } \\ \text { AMY } & \text { Amygdala } \\ \text { Th } & \text { Thalamus }\end{array}$

\section{Introduction}

Episodic autobiographical memory (EAM) is a complex cognitive function that involves a specific large-scale network of interacting brain regions to store and later retrieve our lives' experiences. However, the precise mechanisms that enable the specialized brain areas to encode or retrieve EAM remain unclear. Brain oscillations and synchronization within and across large-scale networks facilitate long-distance communication and promote cognitive functions in the healthy human brain (Buzsáki 2006). Particularly, the slower theta $(3-10 \mathrm{~Hz})$ and faster gamma $(>25 \mathrm{~Hz})$ oscillations have been related to memory processes but likely play distinct mechanistic roles. Gamma oscillations have been suggested related to memory-related synaptic changes (Nakazono et al. 2018; Sederberg et al. 2007). Gamma phase synchrony in the neocortex is well-known to facilitate neuronal communication and functional integration thanks to the precise temporal coincidence of spiking activity (Fries 2009), and in turn binds distributed memory representations that are needed to retrieve episodic memories (Düzel et al. 2010; Fell and Axmacher 2011). Theta oscillations are the dominant rhythms recorded in the hippocampus (Buzsaki 2002) and appear to serve as a mechanism of EAM memories constituted of shorter sub-episodes, whose temporal dynamics during recall are supported by the same mechanism as during encoding (Buzsaki 2002; Buzsáki and Moser 2013). Theta oscillations have been associated with both encoding and retrieval of episodic memories (Friese et al. 2013; Osipova et al. 2006; Sederberg et al. 2003).

Generally, theta phase synchrony is supposed to coordinate the coactivation of distant regions whose local neuronal assembly would be synchronized in the gamma range (Fell and Axmacher 2011). This hypothesis can also be seen as cross-frequency phase-amplitude coupling (PAC); the gamma activity would occur at a specific phase of the lowfrequency oscillation (Canolty and Knight 2010; Fell and Axmacher 2011; Florin and Baillet 2015; Sirota et al. 2008; Tort et al. 2008). The low-frequency oscillation here acts as a metronome that coordinates neural activity in the same region or a remote region, making a putative mechanism for long-range communication. Using simultaneous recordings from both the hippocampus and neocortex in behaving rats and mice, Sirota et al. (Sirota et al. 2008) showed that the theta oscillations in the hippocampus modulate gamma oscillations in the neocortex through PAC. Thus, the coupling between hippocampal theta and neocortical gamma oscillations seems to be a critical mechanism supporting the large-scale memory network's interactions (Axmacher et al. 2010; Nyhus and Curran 2010).

Very few human studies to date have explored the complex communication within and between regions involved in EAM. A few recent magnetoencephalography (MEG) studies of personal memories have started to investigate the mechanisms for the organized communication within and between broad networks at different spatiotemporal scales (Fuentemilla et al. 2014; Hebscher et al. 2019). For example, Fuentemilla et al. (Fuentemilla et al. 2014) found selective phase-synchronization in theta frequency between the medial temporal lobe (MTL), medial prefrontal cortex, and precuneus that was higher during EAM than general semantic retrieval. In a follow-up study, Fuentemilla et al. (2018) sought to identify larger-scale patterns in higher frequencies and found neural synchrony, specifically in the gamma frequency range underlying episodic autobiographical recollection. Hebscher et al. (2019) used theta-burst transcranial magnetic stimulation to examine whether interactions between theta and gamma frequencies facilitate the recall of personal memories and found that memory retrieval is supported by MTL-cortical communication mediated by theta phase coupling and theta-gamma PAC. Although such research emphasizes the importance of brain oscillations in memory processing and integration, these studies relied either on seed-based methods calculating the phasesynchrony between a selected region and the rest of the brain, thus missing potential interactions between regions not interacting with the seed, or scalp level connectivity analysis without any consideration of the source-level network reconstruction. Therefore, there is a need to analyze the interactions both at the source level and between all brain regions without selecting only some brain areas based on their coactivation during the EAM processes or a priori knowledge to obtain a complete picture of the interaction between brain regions.

In the present study, we investigated the dynamic interactions between neuronal networks during EAM retrieval, particularly in the gamma and high theta frequencies. We 
focused on the upper range of the theta band $(7-10 \mathrm{~Hz}$; high theta) because, while the frequency of the human hippocampal theta is rather controversial (Jacobs 2014; de la Prida 2019; Miller et al. 2018), Fuentemilla et al. (2014) found that when participants were asked to retrieve personal episodes and elaborate them during $30 \mathrm{~s}$ during MEG recording, the peak theta frequency linked to the MTL-Precuneus-mPFC phase synchronization was above $7 \mathrm{~Hz}$. We assessed the directed phase connectivity and phase-amplitude coupling at play during EAM recollection, leveraging the high-temporal resolution of EEG and the 64 channels dataset reported in (Bréchet et al. 2019). We first computed the average power in high theta and gamma bands in the source space to investigate which parts of the brain were more active during the EAM retrieval than during a control task. We then applied two types of directed connectivity measures, the directed phase lag index (dPLI) (Stam and van Straaten 2012) and the normalized modulation index (nMI) (Özkurt and Schnitzler 2011; Tort et al. 2008) to quantify the coupling at the wholebrain level between 84 regions of interest (ROIs). The dPLI is a measure of phase-synchronization, which is robust to source leakage and analyzes the direction of the information flow. The nMI allows quantifying the PAC between the phase of low-frequency time-series and the amplitude of the high-frequency course.

Following the studies of (Foster et al. 2015, 2012) and (Dastjerdi et al. 2013), we contrasted the brain activity during the EAM retrieval task to a basic arithmetic equation task as a condition devoid of self-referential content, but still requiring attentional focus. The actual meaning of this contrast was thus to compare the electrical activity during an internally and an externally oriented task.

\section{Materials and Methods}

\section{Participants}

15 healthy participants $(30.5 \pm 5.5$ years, 10 female $)$ were included in the study. The dataset was acquired and described in Bréchet et al. 2019. The local ethics committee's institutional review board approved this study, and all participants provided written informed consent prior to the experiment.

\section{Experimental Paradigm}

Participants were instructed to either retrieve episodic, selfrelated memories ("memory condition") or perform arithmetic calculations ("math condition") while undergoing hd-EEG recording. There were 40 trials in total, each trial lasting $30 \mathrm{~s}$. On each trial, a personal photograph (e.g., photo of a participant with a birthday cake) or a calculation (e.g.,
$447-7=$ ) was presented for $3 \mathrm{~s}$, followed by $27 \mathrm{~s}$ period of closed eyes, during which participants either retrieved the EAM or subtracted/added numbers. Participants were asked to retrieve the personal events in as much detail as possible or fully focus on the calculations. At the end of each trial, participants were asked to rate how much they were able to either relive the past self-related events or pay attention to the self-unrelated calculation. The ratings were similar between the memory and math conditions. For more detail on the experimental paradigm, see (Bréchet et al. 2019).

\section{EEG Recording and Preprocessing}

EEG was recorded with a 64-channel BrainAmp EEG system (Brain Products, Munich, Germany) at $5000 \mathrm{~Hz}$. The EEG recording was down-sampled to $500 \mathrm{~Hz}$ and filtered using a zero-phase digital 4th-order Butterworth bandpass filter between 1 and $80 \mathrm{~Hz}$. Infomax-based Independent Component Analysis (ICA) was applied to remove oculomotor, cardiac, and muscle artifacts based on the channels with maximal amplitude, the topography, and time course of the component (as recommended in Jung et al. 2000). Particular attention was devoted to removing long-standing muscle artifacts, which may overlap and contaminate the genuine gamma activity produced by the brain in the same frequency band $(30-80 \mathrm{~Hz})$. In other words, we removed independent components whose topographies were limited to one or two channels and whose time-courses showed strong activity in the gamma band (Jung et al. 2000). Bad electrodes were interpolated using a 3-D spherical spline (Perrin et al. 1989) and the data were re-referenced to the common average reference. Finally, the remaining artifacts were marked and excluded from further analysis. The preprocessing stages were performed using the Fieldtrip Toolbox (Oostenveld et al. 2011).

\section{EEG Source Imaging}

The forward model for source localization, based on realistic head geometry and conductivity data considering skull thickness (Locally Spherical Model with Anatomical Constraints [LSMAC]) (Brunet et al. 2011), was calculated for a standard set of 64-electrode positions co-registered to the Montreal Neurological Institute (MNI) atlas. The inverse solution space consisted of about 5000 point sources equally distributed in gray matter volume. We computed the inverse solution matrix using the linear distributed source localization procedure LAURA (Grave De Peralta Menendez et al. 2004). The lead-field matrix and the inverse matrix were computed using the freely available software Cartool (Brunet et al. 2011; Michel and Brunet 2019)https:/sites.google. com/site/cartoolcommunity/. 


\section{Frequency-Band Power Analyses}

We computed the analytic signal of the EEG traces using the Hilbert transform and projected the real and imaginary parts in the inverse space using the inverse operator to obtain the average power in high theta and gamma bands at each point source for the memory and math conditions. Before the Hilbert transform, the signals were bandpass filtered in either high theta $(7-10 \mathrm{~Hz})$ or gamma $(30-80 \mathrm{~Hz})$ bands. The square of the norm of the resulting three-dimensional complex signal was calculated to obtain a single real power time-course for each point source. A standardization across time was applied for each point source to eliminate activation biases (Bréchet et al. 2019; Michel and Brunet 2019). These values were then averaged across time and trials, resulting in an average power value at each participant's source location.

\section{Whole-Brain Connectivity Analysis}

We used 84 regions of interest (ROIs) to build the connectivity matrices. In Cartool, each source was assigned to one of the ROIs defined by the second version of the AAL atlas (Rolls et al. 2015; Tzourio-Mazoyer et al. 2002). S1 Figure shows the position of the centroid of each ROI in a 3D brain mesh and S1 Table lists the abbreviations and anatomical descriptions of the AAL ROIs.

Notably, the AAL atlas is an anatomical parcellation of the brain based on gyri and sulci rather than functional specificity. Therefore, we used several ROIs to describe functional brain areas. For example, the vmPFC was described as in Gilboa and Marlatte 2017 and McCormick et al. 2018 with the three following ROIs: the medial orbital part of the superior frontal gyrus, gyrus rectus and the subcallosal gyrus. We projected the filtered data in either high theta or gamma bands into the source space to obtain the different ROIs' time-courses. We took the first component of the singular value decomposition computed over all the time-series of every source (independently of their orientation) belonging to a given ROI. This method was described in Rubega et al. 2019 and used in several other studies (Carboni et al. 2019, 2020; Damborská et al. 2020). The singular value decomposition has a sign ambiguity, which can bias directed connectivity measures. To resolve this indeterminacy, we used the method proposed by Bro et al. 2008, which allocates the sign based on the majority of vectors represented by the component. The non-artifacts sections of the trials were concatenated, and the following connectivity measures were calculated.

We used two types of directed connectivity measures to quantify the coupling between ROIs: the directed Phase Lag Index [dPLI] (Stam and van Straaten 2012) and the normalized
Modulation Index [nMI] (Canolty et al. 2006; Özkurt and Schnitzler 2011).

\section{Directed Phase Lag Index}

The dPLI measures the probability that the difference of the instantaneous phases of two signals is positive, i.e., the probability that one signal has its instantaneous phase leading the instantaneous phase of another signal. Formally:

$d p L I_{x y}:=\frac{1}{N} \sum_{t=1}^{N} H\left(\varphi_{x}(t)-\varphi_{y}(t)\right)$

where $\mathrm{H}$ is the Heaviside step function, $\mathrm{N}$ is the number of samples, $\varphi_{x}$ and $\varphi_{y}$ are respectively the instantaneous phase of the time series $\mathrm{x}$ and $\mathrm{y}$ obtained after applying the Hilbert transform. $d P L I_{x y}$ is bounded between 0 and $1 . \mathrm{x}$ is said to phase-lead y when $d P L I_{x y}>0.5$ and phase-lag when $d P L I_{x y}<0.5$. The critical value of 0.5 is not user-defined but obtained by the definition of the dPLI. Let us suppose that there is no consistency in the difference in phase between signals $\mathrm{x}$ and $\mathrm{y}$. The phase difference will randomly be positive (one half of the time) and negative (the other half of the time). As the Heaviside function outputs 1 for positive numbers and 0 for negative numbers, half of the summands will be ones and half zeros resulting in a dPLI of 0.5. This measure was applied to the high theta and gamma filtered timeseries separately. One connectivity matrix of dPLI in high theta and gamma bands was computed for each participant.

\section{Normalized Modulation Index}

The nMI quantifies the phase-amplitude coupling (PAC) between the phase of low-frequency time-series and the amplitude of the high-frequency course. Mathematically:

$n M I_{x y}:=\frac{1}{\sqrt{N}} \frac{\left|\sum_{t=1}^{N} a_{\gamma}(t) e^{i \phi_{h \theta}(t)}\right|}{\sqrt{\sum_{t=1}^{N} a_{\gamma}(t)^{2}}}$

$a_{\gamma}$ is the amplitude of the analytic signal filtered in gamma, $\phi_{h \theta}$ is the instantaneous phase of the analytic signal filtered in high theta, and $\mathrm{N}$ is the number of samples. Note that the direction of the connectivity does not carry the usual causality meaning but rather that the phase of region $\mathrm{A}$ is coupled to the amplitude of region B (i.e., $\mathrm{A} \rightarrow \mathrm{B}$ means that $\phi_{h \theta}^{A}$ is coupled to $\left.a_{\gamma}^{B}\right)$. We investigated both the local $\left(\mathrm{nMI}_{\mathrm{xx}}\right)$ and inter-regional $\left(\mathrm{nMI}_{\mathrm{xy}}\right)$ PAC. One connectivity matrix of $\mathrm{nMI}$ was computed for each participant. 


\section{Statistical Analysis}

\section{Activation Maps}

We applied a two-sided paired t-test to each point source's average power across participants to investigate which parts of the brain were more active during the memory than the math condition at the group level. A cluster-based permutation procedure implemented in FieldTrip (Maris and Oostenveld 2007; Oostenveld et al. 2011) was used to control for multiple comparisons. Clusters are formed by grouping together neighboring point sources whose t-values were higher than the initial threshold. The initial threshold for cluster definition was set to $\mathrm{p}<0.0005$, and the final threshold for significance of the summed $t$ value within clusters was set to $p<0.05$ with 5000 permutations.

\section{Connectivity Networks}

Networks are defined by nodes and links. In this study, the nodes are the ROIs, and the links are the values of the connectivity of each pair of nodes. We used the network-based statistic (NBS) (Zalesky et al. 2010) to compare the connectivity matrices. The connectivity matrices were subjected first to a t-test and then to a connected-component-based permutation procedure to control for multiple comparisons. In graph theory, a connected component is a set of nodes where any two nodes (e.g., A and B) are connected either directly (A-B) or indirectly (i.e., via other nodes: A-C-D-B). As for the cluster-based statistics, one can derive a measure for each component present after the first level threshold, presently the number of links, and test this measure against values obtained after permutation. Importantly, NBS only works for undirected networks as it uses a breadth-first search (Hopcroft and Tarjan 1973). We modified this part to work with a directed network by searching for weakly connected components, i.e., seeking connected components while ignoring link direction as implemented in Matlab's conncomp. We chose weakly over strongly connected components because strongly connected components have a stricter definition, which may not be realistic in terms of brain networks. A component is defined as strongly connected if and only if for every pair of nodes, there is a directed path between any two nodes. For instance, $\mathrm{A} \rightarrow \mathrm{B} \rightarrow \mathrm{C}$ is weakly connected because there is no directed path to go from $\mathrm{B}$ or $\mathrm{C}$ to $\mathrm{A}$, while $\mathrm{A} \rightarrow \mathrm{B} \rightarrow \mathrm{C} \rightarrow \mathrm{A}$ is strongly connected, as there is a directed path that connects $\mathrm{B}$ to $\mathrm{A}(\mathrm{B} \rightarrow \mathrm{C} \rightarrow \mathrm{A})$ and $\mathrm{C}$ to $\mathrm{A}(\mathrm{C} \rightarrow \mathrm{A})$.

As proposed by Zalesky and colleagues (Zalesky et al. 2010), the data can be transformed prior to the t-test to ensure a meaningful first level threshold. Both the nMI and dPLI are bounded by 0 and 1 . Therefore we applied a logit transform. The logit transform is a monotonically increasing function and transforms values from $(0,1)$ to $(-\infty,+\infty)$, specifically $\operatorname{logit}(0.5)=0$. The above inequalities relative to the dPLI are conserved except that the critical value became 0 instead of 0.5 .

We directly contrasted the memory and math conditions through a paired t-test as the nMI matrices are non-symmetric $\left(n M I_{x y} \neq n M I_{y x}\right)$. The permutations were obtained by randomly exchanging the conditions within subjects. However, the dPLI matrices are anti-symmetric $\left(d P L I_{x y}=-d P L I_{y x}\right)$ and a direct comparison of the conditions is thus not possible; we tested whether the direction of the links were consistent across participants in each condition separately. Therefore, we applied a one-sample t-test against 0 to determine the presence of a consistent link direction between nodes across participants. Here the permutations were obtained by randomly transposing the matrices, i.e., changing the direction of the links. The initial threshold for connected-component definition was set to $p<0.005$. The final threshold for significance of the number of connections within connected components was set to $\mathrm{p}<0.05$ with 5000 permutations. We also defined three different types of nodes: (a) source nodes are nodes with only outgoing links, (b) sink nodes with only incoming links, and (c) intermediate nodes have incoming and outgoing links. When describing the significant networks, we will give their number of links and nodes and, for the sake of readability, only detail the connectivity of their strongest nodes, i.e., nodes with more than three links. The complete networks are shown in the respective figures and supplementary tables.

\section{Comparison of the Distance Between Connected Regions}

Finally, we calculated the distance between the centroids of pairs of connected ROIs for each connectivity type. We performed a one-way ANOVA to test whether there exists a difference in these profiles of distance. Post hoc t-tests were used to explore differences between all the pairs of connectivity types and were controlled for multiple comparisons by the Dunn-Sidák approach (Šidák 1967). All reported p-values were corrected for multiple comparisons by one of the above approaches.

\section{Results}

\section{Power Changes in Source Space}

We found several statistically significant clusters of point sources in gamma and high theta bands when comparing the power in the memory condition to the control/math condition (Fig. 1, Table 1). Specific to the gamma frequency, we found 5 significant clusters (Fig. 1a, Table 1a). The first 


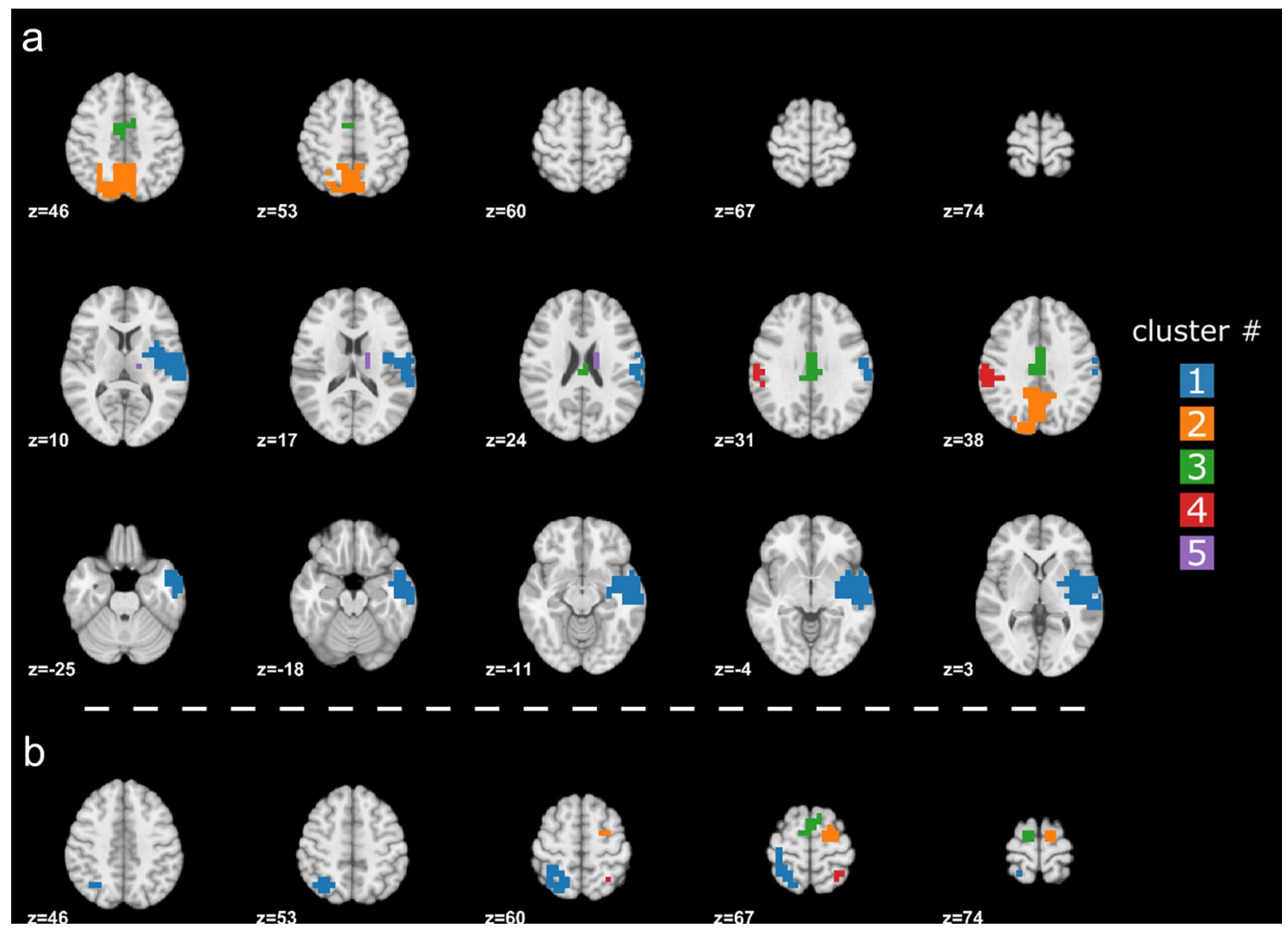

Fig. 1 Significant clusters in a. gamma and b. high theta band when contrasting the memory vs. math conditions. Only slices containing significant clusters are shown. The different clusters are color-coded. Numbers identify the different clusters and are detailed in Table 1

cluster (sum of t: 982.03, $\mathrm{p}<0.01$ ) had its maximum t-value located in the right superior temporal gyrus (STG), and the right temporal pole and supramarginal gyrus (SMG) were its anterior and posterior limits, respectively. It also included part of the right middle temporal gyrus (MTG), insula, rolandic operculum, and postcentral gyrus. The second cluster (sum of t: 624.44, p <0.01) showed a maximum t-value in the left precuneus ( $\mathrm{PrCu}$ ) and included the $\mathrm{PrCu}$ bilaterally and the left superior parietal (SPG) and inferior parietal gyri (IPG). The third cluster (sum of t: 258.80, $\mathrm{p}<0.01$ ) bilaterally included the median cingulate cortex (MCC) and posterior superior frontal gyrus (pSFG), and its peak was in the left MCC. The peak of the fourth cluster was found in the left SMG, and this cluster (sum of t: 89.88, p <0.01) was composed of the left SMG, IPG, and postcentral gyrus. The fifth cluster (sum of t: $32.29, \mathrm{p}<0.05$ ) included the right caudate nucleus and thalamus with a peak in the right caudate nucleus.

We identified 4 significant clusters in the high theta frequency (Fig. 1b, Table 1b). The first cluster (sum of t: $204.54, p<0.01$ ) had its maximum $\mathrm{t}$-value located in the left SPG and included the left postcentral gyrus, IPG, precentral gyrus, and the left $\operatorname{PrCu}$. The second cluster (sum of $\mathrm{t}$ : $60.62, p<0.01$ ) showed a peak in the right superior frontal gyrus (SFG) and included the right pSFG. The third cluster (sum of t: $56.78, \mathrm{p}<0.01$ ) bilaterally included part of the pSFG and the left SFG with a peak in the left pSFG. The fourth cluster (sum of $t: 18.87, p<0.05$ ) showed a maximum $\mathrm{t}$-value in the right SPG and partially included the postcentral gyrus.

\section{Directed Functional Connectivity}

As shown in Fig. 2 and S2 Table, the gamma band directed connectivity analysis revealed a network $(\mathrm{p}<0.01)$ composed of 16 links and 16 nodes (including 9 source, 1 intermediate, and 6 sink nodes). No statistically significant network was found in the math condition, and therefore no link was removed in the network found in the memory condition as described in the Method section. Most of the links connected nodes in the frontoparietal midline and left MTL. Particularly, the amygdala (AMY) was the sink node with the most incoming links (4 links) originating from the hippocampus (HIPP), parahippocampal gyrus (PHG), thalamus (Th), and left PCC. The right subcallosal gyrus (SCG) was the second strongest sink node with 3 links connected to the right PCC, pSFG, and left Th. With the same number of incoming links, the left anterior cingulate cortex (ACC) 
Table 1 Anatomical description of the statistically significant clusters obtained when contrasting memory and math condition in the a. gamma band and $b$. high theta band

\begin{tabular}{|c|c|c|c|c|c|c|c|c|}
\hline $\begin{array}{l}\text { \# Cluster } \\
\text { a. Gamma }\end{array}$ & \multicolumn{7}{|c|}{ a. Gamma band } & $\mathrm{Z}$ \\
\hline 1 & 193 & 0.0014 & $\begin{array}{l}\text { R STG } \\
\text { R MTG } \\
\text { R insula } \\
\text { R rolandic operculum } \\
\text { R PostCG } \\
\text { R Putamen } \\
\text { R SMG } \\
\text { R STGpole } \\
\text { R MTGpole } \\
\text { R heschl gyrus } \\
\text { R ITG } \\
\text { R Pallidum }\end{array}$ & R STG & 6.51 & 55 & -3 & -3 \\
\hline 2 & 128 & 0.0032 & $\begin{array}{l}\text { L precuneus } \\
\text { R precuneus } \\
\text { L SPG } \\
\text { L IPG } \\
\text { L SOG } \\
\text { L MCC } \\
\text { L cuneus } \\
\text { R MCC } \\
\text { L MOG }\end{array}$ & $\mathrm{L}$ precuneus & 5.68 & -3 & -55 & 42 \\
\hline 3 & 54 & 0.0056 & $\begin{array}{l}\text { L MCC } \\
\text { R MCC } \\
\text { L pSFG } \\
\text { R pSFG }\end{array}$ & L MCC & 5.29 & -3 & -10 & 42 \\
\hline 4 & 19 & 0.0090 & $\begin{array}{l}\text { L SMG } \\
\text { L IPG } \\
\text { L PostCG }\end{array}$ & L SMG & 5.27 & -62 & -29 & 36 \\
\hline 5 & 7 & 0.0130 & $\begin{array}{l}\text { R Caudate } \\
\text { R Thalamus }\end{array}$ & R Caudate & 4.70 & 16 & -3 & 23 \\
\hline \multicolumn{9}{|c|}{ b. High theta band } \\
\hline \# Cluster & size & $\mathrm{p}$ & AAL regions & peak region & peak t & $X$ & $\mathrm{Y}$ & $\mathrm{Z}$ \\
\hline 1 & 37 & 0.0030 & $\begin{array}{l}\text { L SPG } \\
\text { L PostCG } \\
\text { L IPG } \\
\text { L precuneus } \\
\text { L PreCG }\end{array}$ & L SPG & 7.10 & -36 & -62 & 62 \\
\hline 2 & 13 & 0.0078 & $\begin{array}{l}\text { R SFG } \\
\text { R pSFG }\end{array}$ & R SFG & 4.89 & 29 & -3 & 68 \\
\hline 3 & 12 & 0.0080 & $\begin{array}{l}\text { L pSFG } \\
\text { R pSFG } \\
\text { L SFG }\end{array}$ & L pSFG & 5.05 & -10 & -3 & 74 \\
\hline 4 & 4 & 0.0120 & $\begin{array}{l}\text { R SPG } \\
\text { R PostCG }\end{array}$ & R SPG & 5.12 & 29 & -55 & 68 \\
\hline
\end{tabular}

The lists of AAL regions included in a cluster are sorted in a descending order according to their number of point sources within the cluster. Sum of t-values correspond to the summation of the t-values within a cluster. The size corresponds to the number of point sources making up the cluster. The region where the peak $\mathrm{t}$-value is found is given under the peak region column. $\mathrm{X}, \mathrm{Y}$ and $\mathrm{Z}$ are MNI coordinates in mm of the peak. Only regions with more than $1 \%$ of their size included in the clusters are listed

Annotations: $L$ left, $R$ Right, $S T G$ superior temporal gyrus, $M T G$ middle temporal gyrus, $I T G$ inferior temporal gyrus, $\operatorname{PreCG}$ precentral gyrus, PostCG postcentral gyrus, $S M G$ supramarginal gyrus, $S P G$ superior parietal gyrus, IPG inferior parietal gyrus, $S O G$ superior occipital gyrus, $M O G$ middle occipital gyrus, $M C C$ median cingulate cortex, $S F G$ superior frontal gyrus, $p S F G$ posterior superior frontal gyrus was connected to the left and right pSFG and the medial orbital part of the right SFG (SFGmedOrb). The pSFG was the source node with the most outgoing links, connecting the ACC and right SCG. 
a

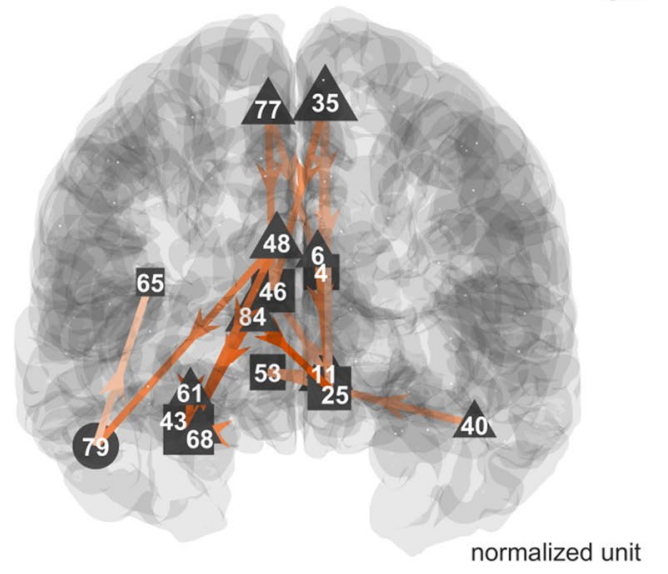

dPLI
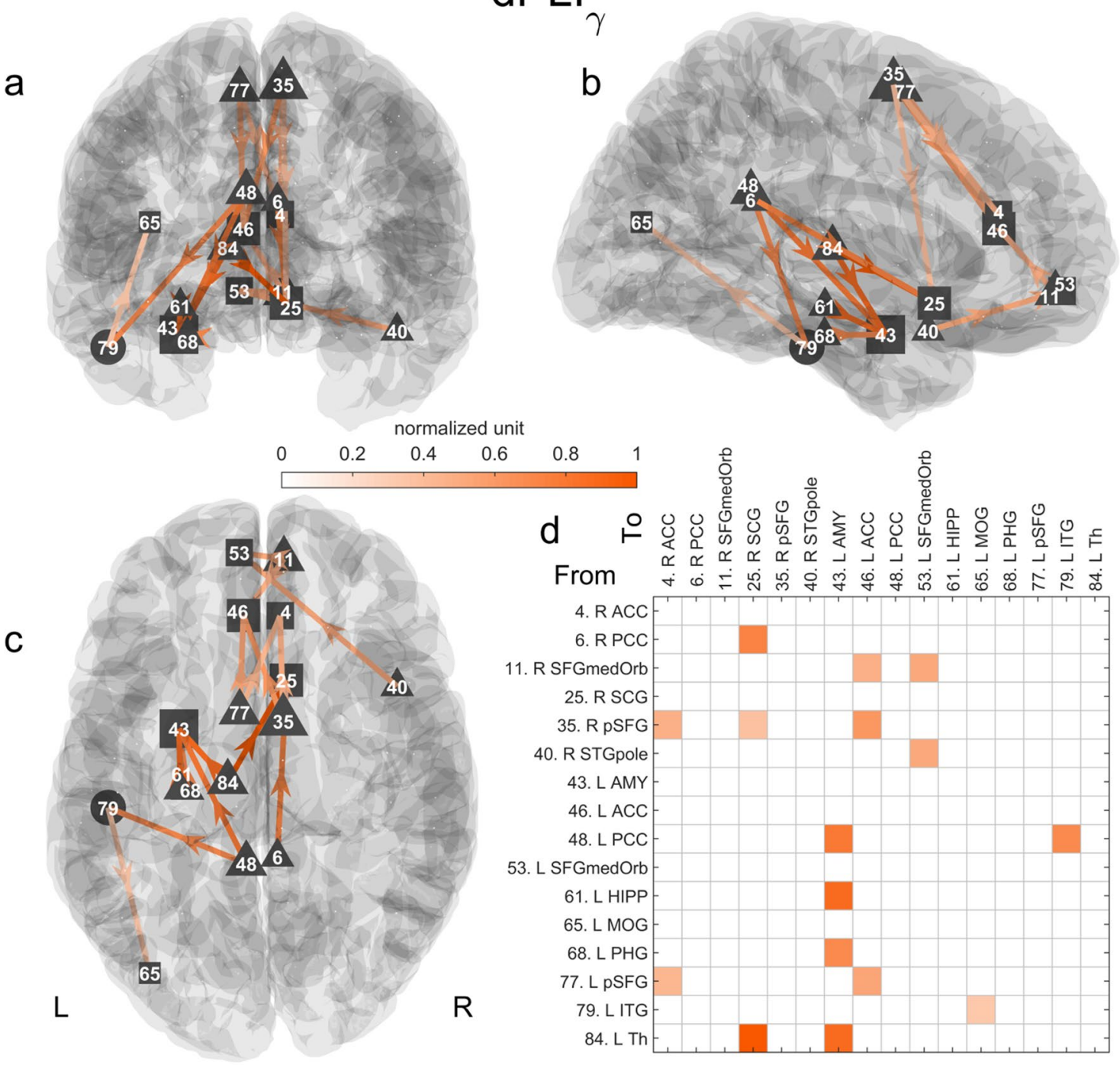

$\mathrm{R}$

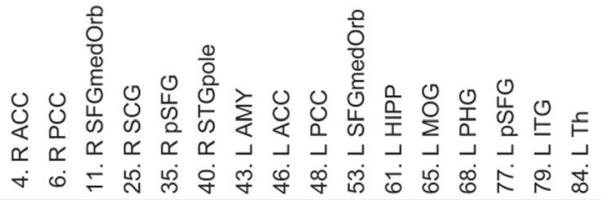

From

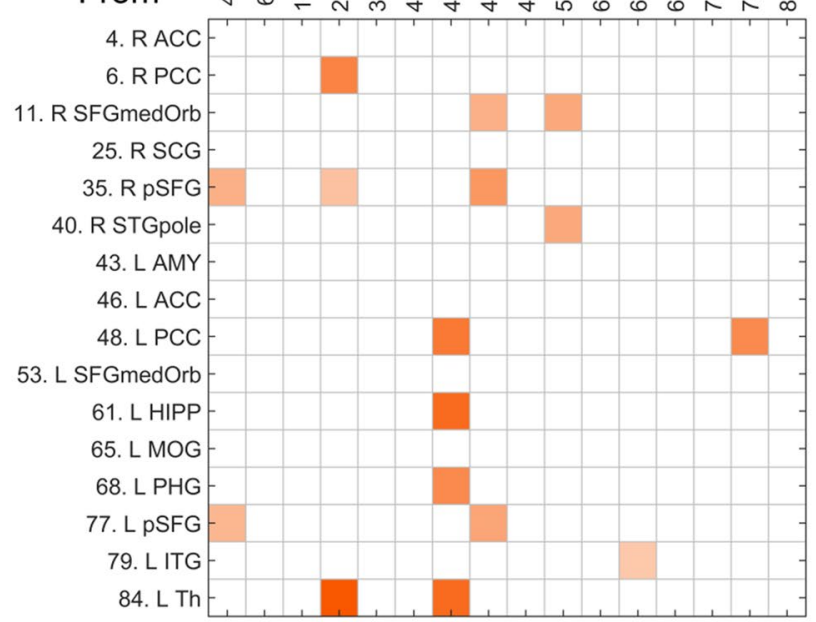

Fig. 2 Significant directed network in gamma in the memory condition. The statistically significant network (16 links and 16 nodes, $\mathrm{p}<0.01)$ is represented in $3 \mathrm{D}$ along the a coronal, b sagittal and $\mathbf{c}$ axial axes. The connectivity matrix is shown in d. Triangles and squares encode nodes with only outgoing or incoming links, i.e., source or sink nodes, respectively. Circles correspond to intermediate nodes with both incoming and outgoing links. The normalized grand average of the connectivity is color-coded. One corresponds

As shown in Fig. 3 and S3 Table, the high theta directed connectivity analysis revealed the presence of a medial posterior-to-anterior network $(\mathrm{p}<0.01)$ composed of 22 links and 18 nodes (including 4 source and 14 sink nodes). Only one link was present in both memory and math conditions $(\mathrm{R}$ PCC to the left gyrus rectus). The left PCC was the source region with the most outgoing links (9 links). This region drove the right ACC and bilaterally the gyrus rectus ( $\mathrm{Rec})$, SCG, Th, and MCC. The right PCC was driving 6 regions: the left and right Rec, Th and SCG. The right inferior

to the maximum value. The size of the node encodes its number of (outgoing or incoming) links. Annotations: $L$ left, $R$ Right, $T h$ thalamus, $S C G$ subcallosal gyrus, $A M Y$ amygdala, HIPP hippocampus, $P C C$ posterior cingulate cortex, ITG inferior temporal gyrus, $P H G$ parahippocampal gyrus, $S F G$ superior frontal gyrus, $p S F G$ posterior SFG, ACC Anterior cingulate cortex, STGpole polar part of the superior temporal gyrus, SFGmedOrb medial orbital part of the SFG, ITG inferior temporal gyrus, $M O G$ middle occipital gyrus

temporal gyrus (ITG) led the right Rec, SFGmedOrb, opercular part of the IFG, and the orbital part of both SFG. Interestingly, the two strongest source nodes (left and right PCCs) were mostly connected to the nodes of the vmPFC (defined by the SFGmedOrb, Rec, and SCG nodes).

\section{Phase-Amplitude Coupled Network}

Compared to the gamma and high theta directed networks, the direction of the PAC-directed network links carries 


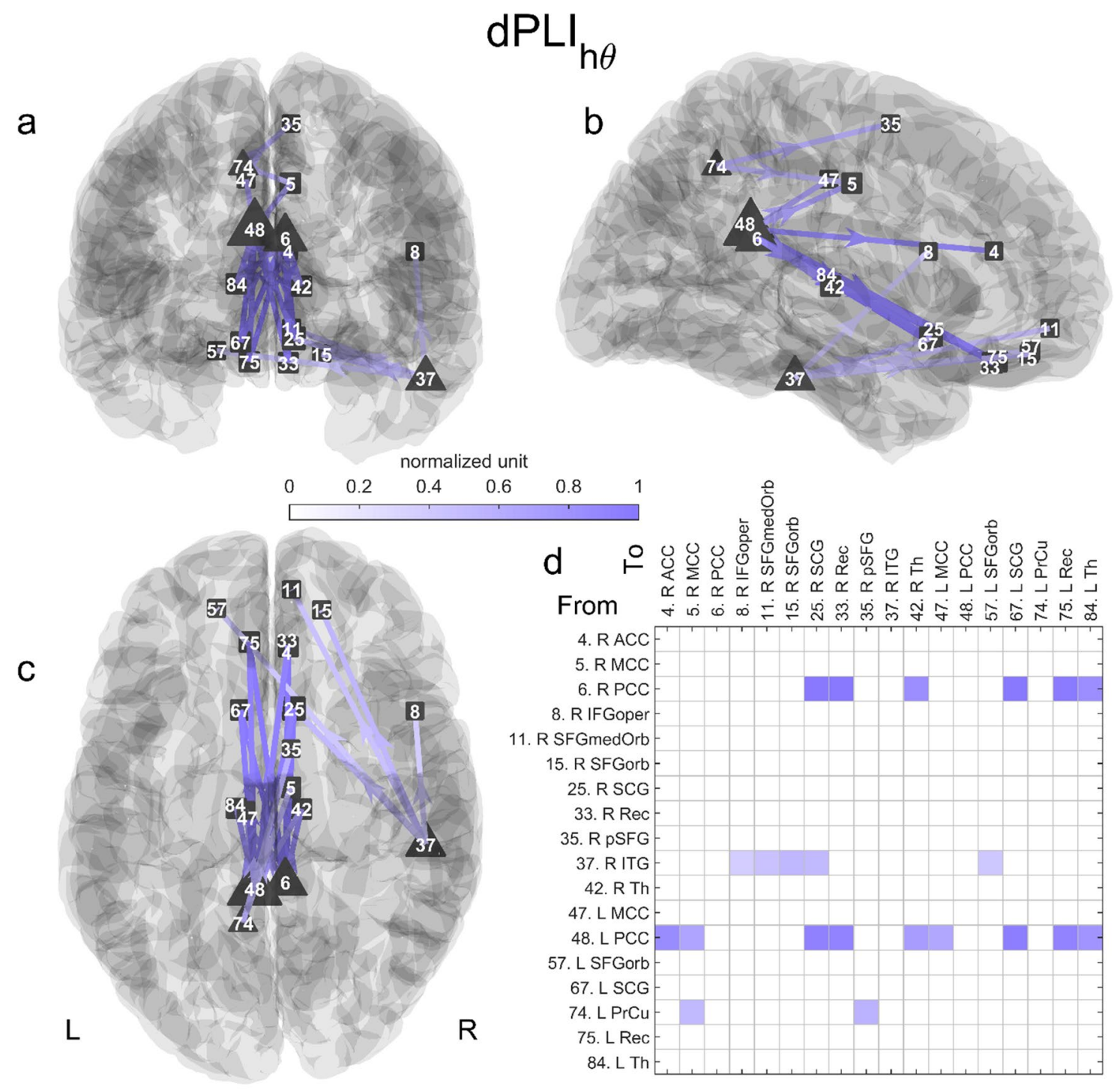

Fig. 3 Significant directed network in high theta in the memory condition. The statistically significant network (22 links and 18 nodes, $\mathrm{p}<0.01)$ is represented in $3 \mathrm{D}$ along the a coronal, b sagittal and $\mathbf{c}$ axial axes. The connectivity matrix is shown in d. Triangles and squares encode nodes with only outgoing or incoming links, i.e., source or sink nodes, respectively. The normalized grand average of the connectivity is color-coded; one corresponds to the maxi-

information about the phase to amplitude relationship. For a given pair of connected nodes, the high theta phase of the node with the outgoing link is coupled to the gamma amplitude of the node with the incoming link. We identified a statistically significant PAC network $(\mathrm{p}<0.05)$ when comparing the memory to the math conditions (Fig. 4 and S4 Table). This network was composed of 20 links and 20 nodes (including 12 source, 3 intermediate, and 5 sink nodes). The left SFGmedOrb (part of the vmPFC) was the sink region with the most incoming links (5 links). Its gamma amplitude was coupled to the high theta phase mum value. The size of the node encodes its number of (outgoing or incoming) links. Annotations: $L$ left, $R$ Right, $P C C$ posterior cingulate cortex, $S C G$ subcallosal gyrus, Rec gyrus rectus, $A C C$ anterior cingulate cortex, Th thalamus, $M C C$ median cingulate cortex, $\mathrm{PrCu}$ precuneus, $S F G$ superior frontal gyrus, $p S F G$ posterior SFG, ITG inferior temporal gyrus, $S F G o r b$ orbital part of the SFG, ITG inferior temporal gyrus, $S F G m e d O r b$ medial orbital part of the SFG

of the left PHG, HIPP, STG, ITG, and lingual gyrus. The right SPG was the second sink node with the most incoming links (4 links). It was coupled to the left HIPP and MCC, and right AMY and superior occipital gyrus. The right Rec (also a node of the vmPFC) had 3 links coming from the right MTG pole, right insula, and left MTG. The left fusiform gyrus received 3 connections from the right STG, precentral gyrus, and angular gyrus. Interestingly, we found that the left HIPP had its high theta phase coupled to gamma amplitude of the two sink nodes with the highest number of links, namely the SFGmedOrb (part of 
a

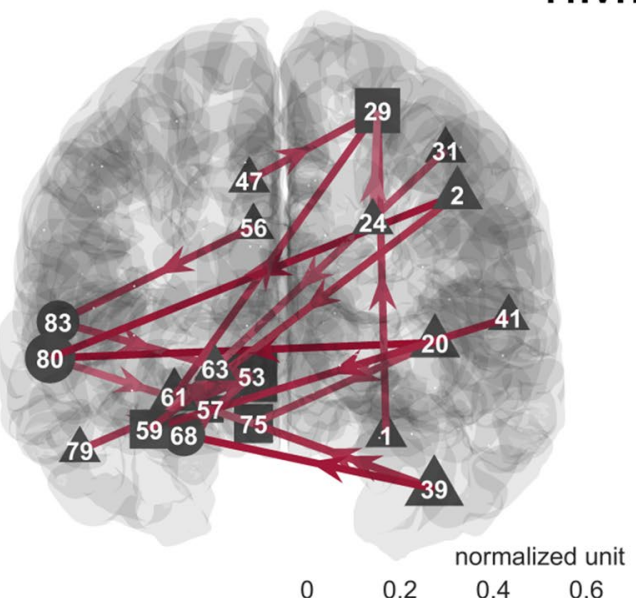

$\mathrm{nMI}$
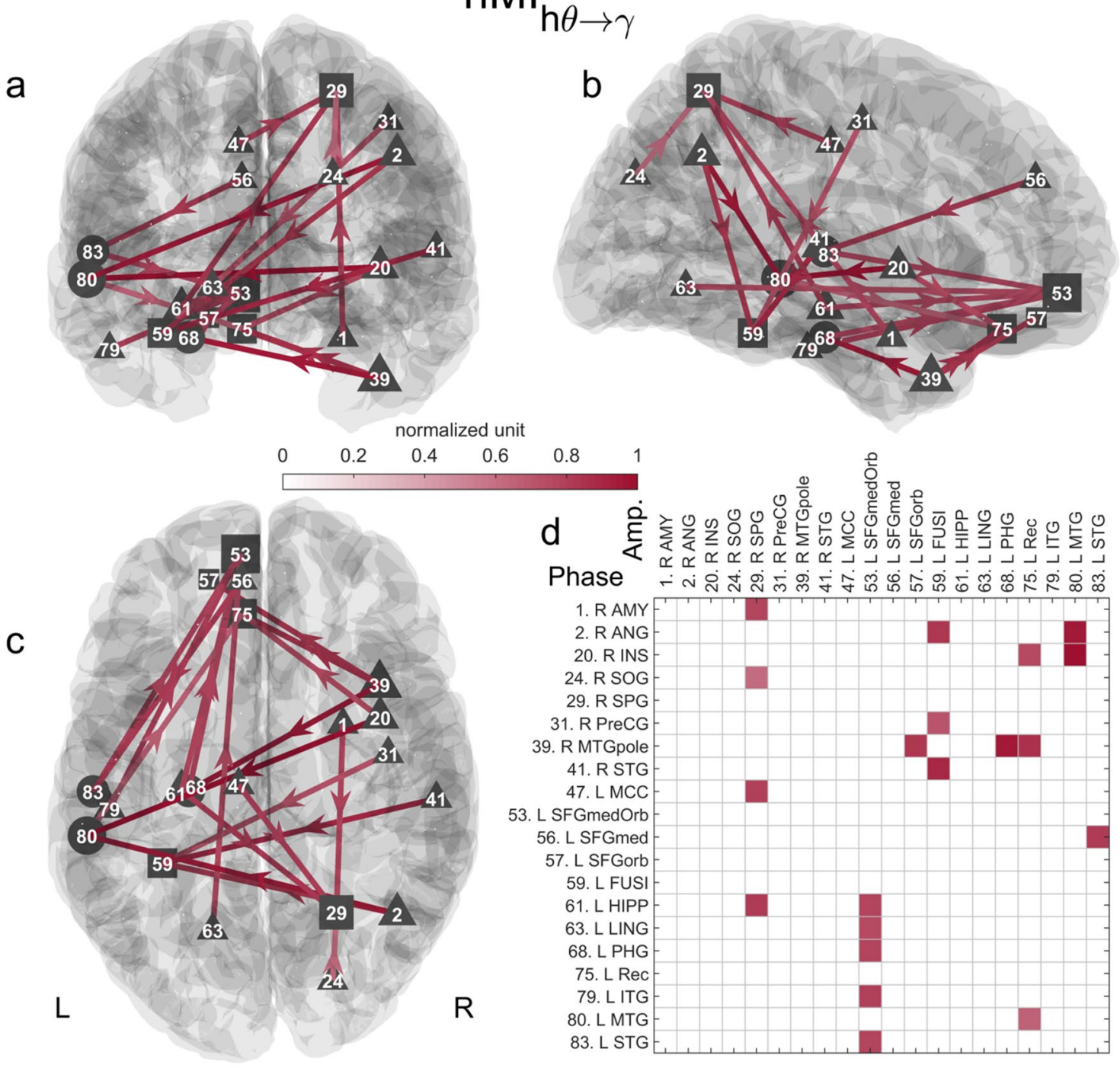

$0.8 \quad 1$

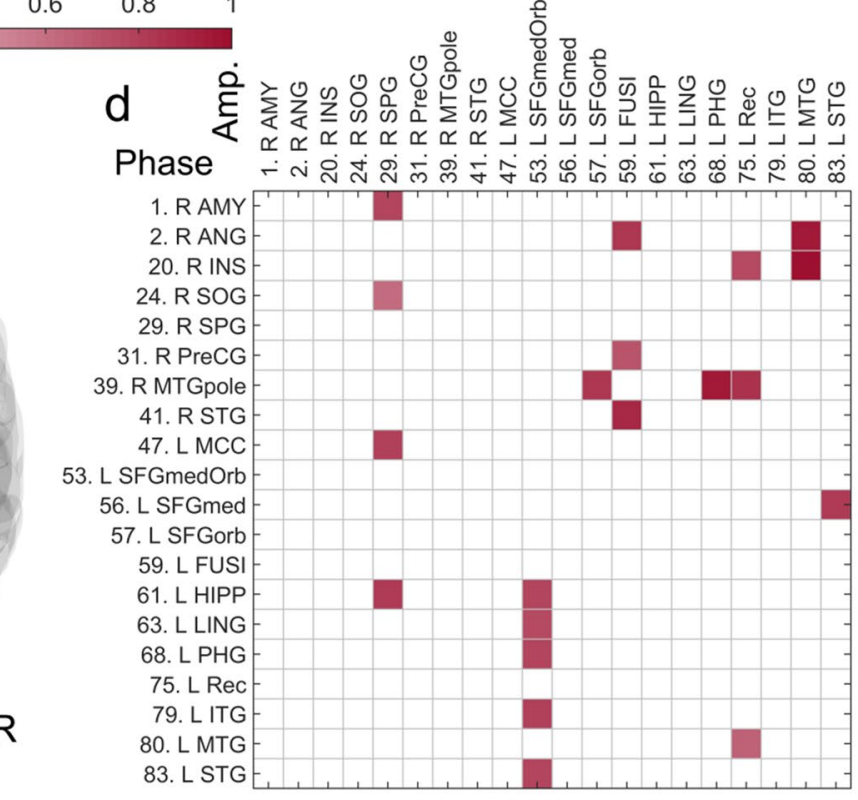

Fig. 4 Significant phase-amplitude coupling directed network when contrasting the memory and math conditions. The statistically significant network (20 links and 20 nodes, $\mathrm{p}<0.05)$ is represented in $3 \mathrm{D}$ along the a coronal, $\mathbf{b}$ sagittal and $\mathbf{c}$ axial axes. The connectivity matrix is shown in d. Triangles and squares encode nodes with only outgoing or incoming links, i.e., source or sink nodes, respectively. Circles correspond to intermediate nodes with both incoming and outgoing links. The normalized grand average of the connectivity is color-coded. One corresponds to the maximum value. The size of the node encodes its number of (outgoing or incoming) links. Anno-

the vmPFC) and right SPG. Notably, the identified PAC network did not contain any local PAC (i.e., phase-amplitude coupling within the same ROI).

\section{ROI to ROI Distance Profile in Different Connectivity Type}

We applied one-way ANOVA to test whether the distributions of the distances between two linked ROIs were different across connectivity types and found a significant result

tations: $L$ left, $R$ Right, INS insula, $M T G$ middle temporal gyrus, $A N G$ angular gyrus, MTGpole polar part of the MTG, $P H G$ parahippocampal gyrus, $S T G$ superior temporal gyrus, FUSI fusiform gyrus, $R e c$ gyrus rectus, $S F G$ superior frontal gyrus, $S F G o r b$ orbital part of the SFG, HIPP hippocampus, SPG superior parietal gyrus, SFGmed medial part of the superior frontal gyrus, $M C C$ median cingulate cortex, ITG inferior temporal gyrus, SFGmedOrb: medial orbital part of the SFG, AMY amygdala, LING lingual gyrus, PreCG precentral cortex, $S O G$ superior occipital gyrus

$(\mathrm{F}=11.32, \mathrm{p}<0.0001$, Fig. 5). Furthermore, the post-hoc t-test revealed that the distances were higher when the ROIs were connected by PAC than by high theta $(p<0.05)$ or gamma phase synchrony $(\mathrm{p}<0.001)$. 


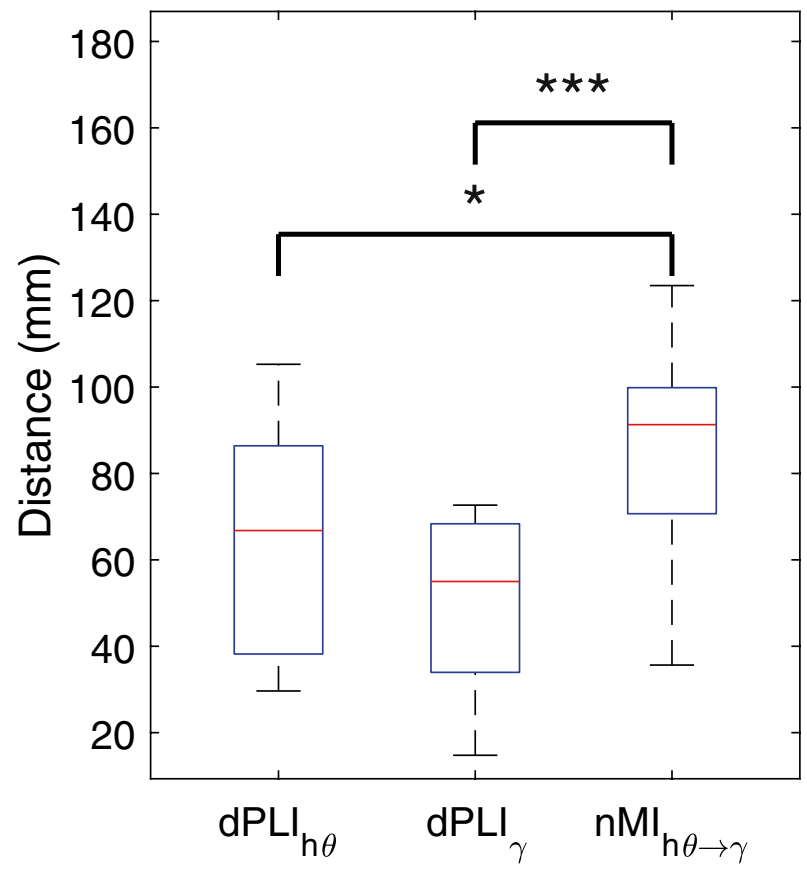

Fig. 5 Boxplot of the distances between ROIs connected either by high theta, gamma phase synchrony, or phase-amplitude coupling. The one-way ANOVA showed that these distributions were not originating from the same distribution $(\mathrm{F}=11.32, \mathrm{p}<0.0001)$. Post-hoc t-tests indicated that the distances between ROIs connected via phaseamplitude coupling (nMI) were longer than via phase synchrony. $(*$ : $\mathrm{p}<0.05, * * *: \mathrm{p}<0.001$, the reported $\mathrm{p}$-value were adjusted after the Dunn-Sidák procedure correcting for multiple comparisons). dPLI: directed phase lag index, nMI: normalized Modulation Index, hy: high theta, $\gamma$ : gamma

\section{Discussion}

\section{A Brief Summary of Main Findings}

Our results showed frequency-specific activation of $\mathrm{PrCu}$, SMG, MCC, and STG in the gamma band and SPG, SFG, and $\mathrm{pSFG}$ in the high theta band. We also found a frequencyspecific directed phase-synchrony network that highlighted the importance of frontoparietal midline and MTL interactions. Specifically, in the gamma band, the left AMY ROI was driven by other MTL regions (HIPP, PHG) and left PCC, and the pSFG, ACC, and vmPFC were interconnected. In the high theta band, a posterior-to-anterior network was found with the PCC driving the vmPFC. Finally, the high theta-gamma PAC network was dense and showed that the MTL phase organized the left vmPFC and right SPG. Overall, the results reveal distinct spatio-temporal signatures in the high theta and gamma bands, with specific interactions that we hypothesize reflect distinct cognitive processes of self-referential autobiographical memory retrieval.

\section{Increased Gamma Activity in Regions Linked to Self-Relevant Processing}

The brain regions with increased gamma activity were primarily found in the left $\mathrm{PrCu}$, left SMG, right STG, and MCC. Generally, these gamma activity clusters seem to occur in regions whose role is related to the first-person perspective and self-orientation.

The $\mathrm{PrCu}$ and the SMG have been implicated in selfrelevant processing. The $\mathrm{PrCu}$ is supposed to shift the frame of reference from an allocentric to an egocentric one (Hebscher et al. 2018), and stimulation of this region has been shown to alter the autobiographical memory retrieval (Hebscher et al. 2020, 2019). In a case report, it was reported that epileptic seizures involving the left SMG provoked autonomic hallucinations (i.e., seeing the double of oneself from an internal point of view) in a patient investigated with intracerebral EEG (Fonti et al. 2020). In addition, these two regions ( $\mathrm{PrCu}$ and $\mathrm{SMG}$ ) were also found in the fMRI analysis in the original paper of the analyzed dataset (Bréchet et al. 2019), strengthening the implication of these regions in EAM.

The MCC is known to be involved in the head and body's reflexive orientation in space (Vogt 2016). This region could work in concert with the PrCu and SMG areas to create an internal representation and sense of self-orientation during EAM recall. The right STG is often listed as activated during recollection of EAM (Boccia et al. 2019; Bréchet et al. 2018) but does not belong to the core regions, unlike the anterior temporal area (Svoboda et al. 2006). Of note, it could be possible that, because of the lower spatial resolution of the EEG, the activation of the right temporal junction and the right anterior temporal area merged into a single cluster.

\section{Activation of the Dorsal Frontoparietal Network in High Theta Band}

Increased activation of the SFG, pSFG, and SPG was found in a high theta band in the memory condition compared to the math condition. Because the clusters found in the SFG do not entirely delineate this gyrus, it can be further interpreted as belonging to the superior frontal sulcus. These regions are known to be part of the dorsal Frontoparietal Network (dFPN) (Petersen and Posner 2012; Ptak et al. 2017). Its functions are diverse and include motor planning and imagery, mental rotation, spatial attention, and working memory (Ptak et al. 2017). At first, it could seem unexpected to find such a visual attentional network more active in the EAM condition than in the math condition. However, during the memory condition, the participants were asked to relive past events depicted on self-related photographs for more than twenty seconds. This requires manipulating and maintaining the scene internally, which is in line with functions 
attributed to the dFPN. Although the math condition is a working memory task, the regions of the dFPN were less involved in that condition. Solving simple arithmetic operations rely more on the frontal network (Ishii et al. 2014). Our previous study showed that one specific microstate occurred more often during the math than memory condition (Bréchet et al. 2019). This microstate's source localization revealed activity in the dorsal ACC, IFG, and IPG, attributed to the executive control system (Ferris and Peterson 2012).

\section{MTL Organizes Local Gamma Synchronization in Prefrontal and Parietal Cortices}

Our PAC result showed that the left hippocampus' high theta phase orchestrated the gamma activity in the medial orbital part of the superior frontal gyrus (belonging to vmPFC) and right superior parietal gyrus. According to the "readerinitiated" mechanism hypothesis (Buzśaki and Wang 2012; Sirota et al. 2008), the hippocampus through theta oscillations can initiate long-range reciprocal communication with neocortical gamma oscillations. In that case, the hippocampus would act as a metronome that coordinates neocortical gamma oscillators. Those gamma oscillations then locally, i.e., within the same region, synchronize to combine information sent back to the hippocampus. We determine that the left AMY node received information from the left PCC, Th, PHG, and HIPP via gamma phase synchrony. Because of the lower spatial resolution of EEG, it might be possible that the AMY node activity globally represents the activity of the anterior MTL rather than activity solely of the AMY. Similarly, we interpret the PHG and HIPP nodes as representing the MTL activity and only refer to their name to describe their connections within the network. This gamma phase synchrony could be the reciprocal connection initiated via PAC by the left MTL with the right superior parietal gyrus, which sends information back via the PCC to the anterior MTL.

A possible role of this reciprocal connection is to perform the high-resolution contextual binding to generate a coherent scene (Yonelinas 2013). Indeed the nodes of this gamma sub-network (AMY, HIPP, PHG, PCC) form the scene-reconstruction network (Andrews-Hanna et al. 2010; Axelrod et al. 2017; Hassabis and Maguire 2007), which was also found in the original paper's BOLD connectivity analysis and EEG microstate analysis (Bréchet et al. 2019). Furthermore, as defined in AAL, the PCC ROI contains part of the retrosplenial cortex (Rolls et al. 2015). This region works in concert with the Th, which sends head direction information, to switch between allocentric and egocentric perspective (Vann et al. 2009). The anterior hippocampus, which is more engaged during scene and events reconstruction than the posterior hippocampus (Zeidman and Maguire 2016), reconstructs the scene from an allocentric viewpoint.
This gamma connectivity from the PCC/retrosplenial cortex, Th, and PHG to anterior MTL could help orient EAM recollection to a personal view.

The second node entrained by the hippocampal phase was in the vmPFC. This node was part of a more extensive gamma phase synchrony sub-network constituted by the ACC, pSFG, and other nodes of the vmPFC. Fuentemilla et al. (2018) showed that large-scale gamma synchrony supports autobiographical recollection and that this synchrony also occurred in the midline frontal MEG sensor, in line with our findings. The putative role of the vmPFC is to monitor memory veracity and correctness (Barry and Maguire 2019; Eichenbaum 2017; Gilboa and Moscovitch 2017; Hebscher and Gilboa 2016; Ritchey and Cooper 2020). This frontal gamma phase synchronization could be the mechanism by which the vmPFC combines information from the prefrontal cortex to verify the retrieved memory's validity.

Finally, when analyzing the distance between the pairs of coupled ROIs, we showed that the distance between ROIs connected via PAC was higher than that via high theta or gamma phase synchronization. PAC thus seems to be the preferred mechanism for long-range coupling, while high theta or gamma phase synchronization appears to be the preferred mechanism for local coupling. Our results suggest that the MTL by entraining with its high theta phase local gamma synchronization in remote and functionally diverse regions, namely the medial prefrontal and the posterior associative cortex, permits to reconstruct a coherent and valid self-relevant episode.

\section{PCC Leads and vmPFC Follows the Network Dynamics}

On the one hand, we found nodes within the vmPFC as sink nodes in the high theta phase synchrony network and the PAC network. This result suggests that the vmPFC is driven via the high theta oscillations in the former network and that gamma activity is coupled to other regions' high theta phase in the latter network. On the other hand, we found the PCC to exclusively contain source nodes in high theta and gamma phase synchrony network, i.e., the PCC was phase leading other regions. Specifically, the network analysis revealed a PCC to vmPFC connection in the high theta band. This result is supported by anatomical connections, namely the cingulum bundle, between these two regions (Bubb et al., 2018) and a recent paper showing the same link in the alpha band within the Default Mode Network (Wang et al. 2019). The PCC is supposed to play a critical role in integrating relevant episodic information emerging from posterior cortices (Andrews-Hanna et al. 2014; Bird et al. 2015). This directed connectivity suggests that once episodic elements are integrated within the PCC, they are sectioned within high theta cycles and sequentially made available via high 
theta phase synchrony to the vmPFC to either maintain or withhold a memory candidate. The high number of outgoing links exhibited by the PCC in the high theta phase synchrony network highlights its central role in EAM. Our study supports with other fMRI- and MEG-based studies that the PCC is a hub for autobiographical and self-referential processing (Andrews-Hanna et al. 2010; Axelrod et al. 2017; de Pasquale et al. 2012; Tang et al. 2017).

Furthermore, the vmPFC's gamma activity was coupled to regions other than the left MTL (as discussed above), including the left STG, ITG, the right MTG pole, and the right insula. These regions are linked to either semantic memory processing or emotion processing and are known to contribute to EAM recollection by adding semantic and emotional features (Svoboda et al. 2006). This PAC relationship suggests that the vmPFC is phase-biased by these regions to constrain the memory validation procedure by the semantic features supporting the EAM.

The fact that the vmPFC was mainly a sink node seems to contradict the results of McCormick et al. (2020). While the memory and baseline conditions are relatively similar, our studies differ in several ways. First, they focused on the five first seconds of the trial while we analyzed the whole trial. Therefore, their finding could describe the construction phase of the EAM while ours encompasses both the construction and elaboration of EAM. Moreover, the hippocampal and vmPFC ROIs in McCormick et al. (2020) were selected by contrasting the memory and math/baseline condition and corresponded to areas with attenuation or decrease of broadband (1-30 Hz) activity. Our ROIs were based on an anatomical atlas. Finally, in McCormick et al. (2020), dynamic causal modelling was used to determine which of the hippocampus or the vmPFC was driving the other regions, which, as far as we know, does not model phase-amplitude coupling or phase synchronization (i.e., the two mechanisms considered in our investigation). Rather than contradicting each other, our study expands the findings of McCormick et al. (2020) and conveys different, complementary information about the dynamic procedure of EAM recall.

\section{Potential Limitations and the Curse of Double-Dipping}

A recent paper from the OHBM COBIDAS committee (Pernet et al. 2020) emphasized the problem of double-dipping (Kriegeskorte et al. 2009, 2010). This problem could arise when secondary statistical analysis (in our case, the connectivity analysis) is applied to ROIs selected with the same statistical criterion using the same dataset. To avoid this issue, we could have selected ROIs with large power in both conditions or with larger power in the memory vs. math condition but using an independent dataset. Rather than selecting ROIs, we avoided double-dipping by applying whole-brain analyses. Interestingly, the regions with high theta or gamma power increase were almost not present in the three obtained networks. The PrCu, SMG, and MCC showed a greater gamma power in the memory than in the math condition, but they were absent in the gamma phase synchrony and PAC network. Likewise, the SPG and SFG were absent in the high theta phase network while showing a high theta power increase. The results would have been strikingly different if only the activated ROIs had been selected for the network analysis. In fact, ROIs with higher activity are not necessarily connected to each other as their activity could evolve independently of each other, especially in our case with trials lasting several tens of seconds. Moreover, brain areas that do not exhibit a difference in power between the conditions could be differently connected in the two conditions. These interactions would be missed if connectivity analysis is applied only to ROIs with larger activity in the condition of interest. We thus believed that applying wholebrain connectivity analysis, as proposed here, is more appropriate to capture networks completely.

PAC integrates the gamma band's amplitude and thus could be biased by changes in amplitude. However, the regions whose gamma activity is synchronized to the high theta phase of another region are not present in the gamma's activation map, especially the vmPFC. Therefore, the PAC network cannot be uniquely attributed to differences in gamma power between the conditions.

Furthermore, gamma activity in scalp EEG can be highly influenced by noise or muscle artifacts. If such artifacts were still present after ICA denoising, they would generate zerolag connectivity between regions affected by the artefact. As the dPLI is insensitive to zero-lag connectivity and no self-loop, i.e., a region is connected to itself, is present in the PAC network, it is unlikely that artifact bias the obtained networks.

Finally, the EEG system used only recorded signals from 64 electrodes while we studied 84 ROIs. The time-courses of the ROIs were not independent. The connectivity measures used in this study are however not sensitive to leakage and therefore not sensitive to correlated ROI time-courses. Moreover, the networks found only contained few ROIs $(\leq 20)$ which is expected with 64 EEG channels. We acknowledge that more refined networks or more networks could have been found with an EEG system with more channels.

\section{Conclusion}

In sum, our findings suggest that the left medial temporal lobe (Hipp, AMG and PHG), the ventromedial prefrontal cortex (vmPFC), and parietal regions (PCC and SPG) work in tandem during recall of self-relevant episodic memories. Importantly, this synergy occurs thanks to complex 
coordination via frequency-specific and cross-frequency coupling. The medial temporal lobe orchestrates the gamma activity in the ventromedial prefrontal cortex and parietal regions with its high theta phase. These two areas synchronize in phase their gamma activity with neighboring regions to, possibly, valuate and construct the memory. Finally, a high theta synchronization occurs between the posterior cingulate cortex and medial prefrontal regions, which could show the transfer of sub-episodes of memory from posterior to frontal regions. Future studies should investigate these relationships utilizing a higher number of electrodes and a paradigm allowing the separation of the activity of regions related to semantic memory to refine these episodic autobiographical memory networks further.

Supplementary Information The online version contains supplementary material available at https://doi.org/10.1007/s10548-022-00890-4.

Funding Open access funding provided by University of Geneva. This work was supported by the Swiss National Science Foundation to LB (Grant No. 187949) and CM (Grant No. 320030_184677), and by the National Center of Competence in Research (NCCR) 'SYNAPSYThe Synaptic Bases of Mental Diseases' financed by the Swiss National Science Foundation (SNF, Grant No. 51NF40 - 185897).

Data Availability The participants informed consent form did not include permission to allow data to be uploaded to an online repository. Data and materials are available upon direct request and are subject to anonymization to protect the privacy of participants. Requestors must sign a formal data sharing agreement.

Code Availability Data were analyzed using open source and open access tools. EEG recording were analyzed using Fieldtrip (Oostenveld et al. 2011), available at: https://github.com/fieldtrip/fieldtrip. The lead-field matrix and the inverse matrix were computed using the freely available software Cartool (Brunet et al. 2011; Michel and Brunet 2019); available at https://sites.google.com/site/cartoolcommunity/. The Network Based Statistic toolbox(Zalesky et al. 2010) is available at https://www.nitrc.org/projects/nbs.

Open Access This article is licensed under a Creative Commons Attribution 4.0 International License, which permits use, sharing, adaptation, distribution and reproduction in any medium or format, as long as you give appropriate credit to the original author(s) and the source, provide a link to the Creative Commons licence, and indicate if changes were made. The images or other third party material in this article are included in the article's Creative Commons licence, unless indicated otherwise in a credit line to the material. If material is not included in the article's Creative Commons licence and your intended use is not permitted by statutory regulation or exceeds the permitted use, you will need to obtain permission directly from the copyright holder. To view a copy of this licence, visit http://creativecommons.org/licenses/by/4.0/.

\section{References}

Andrews-Hanna JR, Reidler JS, Sepulcre J, Poulin R, Buckner RL (2010) Functional-anatomic fractionation of the brain's default network. Neuron 65(4):550-562. https://doi.org/10.1016/j.neuron. 2010.02.005

Andrews-Hanna JR, Saxe R, Yarkoni T (2014) Contributions of episodic retrieval and mentalizing to autobiographical thought: evidence from functional neuroimaging, resting-state connectivity, and fmri meta-analyses. Neuroimage 91:324-335. https://doi.org/ 10.1016/j.neuroimage.2014.01.032

Axelrod V, Rees G, Bar M (2017) The default network and the combination of cognitive processes that mediate self-generated thought. Nat Hum Behav 1(12):896-910. https://doi.org/10.1038/ s41562-017-0244-9

Axmacher N, Henseler MM, Jensen O, Weinreich I, Elger CE, Fell J (2010) Cross-frequency coupling supports multi-item working memory in the human hippocampus. Proc Natl Acad Sci USA 107(7):3228-3233. https://doi.org/10.1073/pnas.0911531107

Barry DN, Maguire EA (2019) Remote memory and the hippocampus: a constructive critique. Trends Cogn Sci 23(2):128-142. https:// doi.org/10.1016/j.tics.2018.11.005

Bird CM, Keidel JL, Ing LP, Horner AJ, Burgess N (2015) Consolidation of complex events via reinstatement in posterior cingulate cortex. J Neurosci 35(43):14426-14434. https://doi.org/10.1523/ JNEUROSCI.1774-15.2015

Boccia M, Teghil A, Guariglia C (2019) Looking into recent and remote past: meta-analytic evidence for cortical re-organization of episodic autobiographical Memories. Neurosci Biobehav Rev 107(March):84-95. https://doi.org/10.1016/j.neubiorev.2019.09. 003

Bréchet L, Grivaz P, Gauthier B, Blanke O (2018) Common recruitment of angular gyrus in episodic autobiographical memory and bodily self-consciousness. Front Behav Neurosci 12:1-10. https:// doi.org/10.3389/fnbeh.2018.00270

Bréchet L, Brunet D, Birot G, Gruetter R, Michel CM, Jorge J (2019) Capturing the spatiotemporal dynamics of self-generated, taskinitiated thoughts with EEG and FMRI. Neuroimage 194:82-92. https://doi.org/10.1016/j.neuroimage.2019.03.029

Bro R, Acar E, Kolda TG (2008) Resolving the sign ambiguity in the singular value decomposition. J Chemom 22(2):135-140. https:// doi.org/10.1002/cem.1122

Brunet D, Murray MM, Michel CM (2011) Spatiotemporal Analysis of Multichannel EEG: CARTOOL. Comput Intell Neurosci. https:// doi.org/10.1155/2011/813870

Bubb EJ, Metzler-Baddeley C, Aggleton JP (2018) The cingulum bundle: anatomy, function, and dysfunction. Neurosci Biobehav Rev 92:104-127. https://doi.org/10.1016/j.neubiorev.2018.05.008

Buzsaki G (2002) Theta oscillations in the hippocampus. Neuron 33:325-340

Buzsáki G, Moser EI (2013) Memory, navigation and theta rhythm in the hippocampal-entorhinal system. Nat Neurosci 16(2):130-138. https://doi.org/10.1038/nn.3304

Buzśaki G, Wang XJ (2012) Mechanisms of Gamma Oscillations. Annu Rev Neurosci 35:203-225. https://doi.org/10.1146/annur ev-neuro-062111-150444

Buzsáki, György. 2006. Rhythms of the Brain.

Carboni, Margherita, Pia De Stefano, Bernd J. Vorderwülbecke, Sebastien Tourbier, Emeline Mullier, Maria Rubega, Shahan Momjian, Karl Schaller, Patric Hagmann, Margitta Seeck, Christoph M. Michel, Pieter van Mierlo, and Serge Vulliemoz. 2020. "Abnormal Directed Connectivity of Resting State Networks in Focal Epilepsy." NeuroImage: Clinical 27: 1023 doi: https://doi. org/10.1016/j.nicl.2020.102336.

Canolty RT, Knight RT (2010) The Functional role of cross-frequency coupling. Trends Cogn Sci 14(11):506-515. https://doi.org/10. 1016/j.tics.2010.09.001

Canolty RT, Barbaro NM, Edwards E, Kirsch HE, Dalal SS, Nagarajan SS, Knight RT, Berger MS, Soltani M (2006) High Gamma power 
is phase-locked to theta oscillations in human neocortex. Science 313(5793):1626-1628. https://doi.org/10.1126/science.1128115

Carboni M, Rubega M, Iannotti GR, De Stefano P, Toscano G, Tourbier S, Pittau F, Hagmann P, Momjian S, Schaller K, Seeck M, Michel CM, van Mierlo P, Vulliemoz S (2019) The network integration of epileptic activity in relation to surgical outcome. Clin Neurophysiol 130(12):2193-2202. https://doi.org/10.1016/j.clinph. 2019.09.006

Damborská A, Honzírková E, Barteček R, Hořínková J, Fedorová S, Ondruš Š, Michel CM, Rubega M (2020) Altered directed functional connectivity of the right amygdala in depression: highdensity EEG Study. Sci Rep 10(1):1-14. https://doi.org/10.1038/ s41598-020-61264-z

Dastjerdi M, Ozker M, Foster BL, Rangarajan V, Parvizi J (2013) Numerical processing in the human parietal cortex during experimental and natural conditions. Nat Commun. https://doi.org/10. 1038/ncomms 3528

de la Prida L, Menendez. (2019) The Theta Stone: 3 to $10 \mathrm{~Hz}$ oscillations to decipher the human brain code. Epilepsy Currents 19(2):126-128. https://doi.org/10.1177/1535759719835662

de Pasquale F, Penna SD, Snyder AZ, Marzetti L, Pizzella V, Romani GL, Corbetta M (2012) A Cortical core for dynamic integration of functional networks in the resting human brain. Neuron 74(4):753-764. https://doi.org/10.1016/j.neuron.2012.03.031

De Peralta G, Menendez R, Murray MM, Michel CM, Martuzzi R, Gonzalez SL, Andino. (2004) Electrical neuroimaging based on biophysical constraints. Neuroimage 21(2):527-539. https://doi. org/10.1016/j.neuroimage.2003.09.051

Düzel E, Penny WD, Burgess N (2010) Brain oscillations and memory. Curr Opin Neurobiol 20(2):143-149. https://doi.org/10.1016/j. conb.2010.01.004

Eichenbaum H (2017) Prefrontal-hippocampal interactions in episodic memory. Nat Rev Neurosci 18(9):547-558. https://doi.org/10. 1038/nrn.2017.74

Fell J, Axmacher N (2011) The role of phase synchronization in memory processes. Nat Rev Neurosci 12(2):105-118. https://doi.org/ 10.1038/nrn2979

Ferris DP, Peterson SM (2012) Combined head phantom and neural mass model validation of effective connectivity measures. $\mathbf{J}$ Acquir Immune Defic Syndr 59(2):593-600. https://doi.org/10. 1097/QAI.0b013e31823e2278

Florin E, Baillet S (2015) The Brain's Resting-State Activity Is Shaped by Synchronized Cross-Frequency Coupling of Neural Oscillations. Neuroimage 111:26-35. https://doi.org/10.1016/j.neuro image.2015.01.054

Fonti D, Lagarde S, Scholly J, Lépine A, Scavarada D, Puligheddu M, McGonigal A, Bartolomei F (2020) Anatomical electroclinical Correlations during an SEEG-recorded seizure with autoscopic hallucination. Epileptic Disorders : International Epilepsy Journal with Videotape 22(6):817-822. https://doi.org/10.1684/epd. 2020.1228

Foster BL, Dastjerdi M, Parvizi J (2012) Neural Populations in human posteromedial cortex display opposing responses during memory and numerical processing. Proc Natl Acad Sci USA 109(38):15514-15519. https://doi.org/10.1073/pnas.1206580109

Foster BL, Rangarajan V, Shirer WR, Parvizi J (2015) Intrinsic and task-dependent coupling of neuronal population activity in human parietal cortex. Neuron 86(2):578-590. https://doi.org/10.1016/j. neuron.2015.03.018

Fries P (2009) neuronal gamma-band synchronization as a fundamental process in cortical computation. Annu Rev Neurosci 32:209-224. https://doi.org/10.1146/annurev.neuro.051508.135603

Friese U, Köster M, Hassler U, Martens U, Trujillo-Barreto N, Gruber T (2013) Successful memory encoding is associated with increased cross-frequency coupling between frontal theta and posterior gamma oscillations in human scalp-recorded EEG.
Neuroimage 66:642-647. https://doi.org/10.1016/j.neuroimage. 2012.11.002

Fuentemilla L, Barnes GR, Düzel E, Levine B (2014) Theta Oscillations Orchestrate Medial Temporal Lobe and Neocortex in Remembering Autobiographical Memories. Neuroimage 85:730737. https://doi.org/10.1016/j.neuroimage.2013.08.029

Fuentemilla L, Palombo DJ, Levine B (2018) Gamma phase-synchrony in autobiographical memory: evidence from magnetoencephalography and severely deficient autobiographical memory. Neuropsychologia 110:7-13. https://doi.org/10.1016/j.neuropsychologia. 2017.08.020

Gilboa A, Marlatte H (2017) Neurobiology of schemas and schemamediated memory. Trends Cogn Sci 21(8):618-631. https://doi. org/10.1016/j.tics.2017.04.013

Gilboa A, Moscovitch M (2017) Ventromedial prefrontal cortex generates pre-stimulus theta coherence desynchronization: a schema instantiation hypothesis. Cortex 87:16-30. https://doi.org/10. 1016/j.cortex.2016.10.008

Hassabis D, Maguire EA (2007) Deconstructing episodic memory with construction. Trends Cogn Sci 11(7):299-306. https://doi.org/10. 1016/j.tics.2007.05.001

Hebscher M, Gilboa A (2016) A Boost of Confidence: the role of the ventromedial prefrontal cortex in memory, decision-making, and schemas. Neuropsychologia 90:46-58. https://doi.org/10.1016/j. neuropsychologia.2016.05.003

Hebscher M, Levine B, Gilboa A (2018) The precuneus and hippocampus contribute to individual differences in the unfolding of spatial representations during episodic autobiographical memory. Neuropsychologia 110:123-133. https://doi.org/10. 1016/j.neuropsychologia.2017.03.029

Hebscher M, Meltzer JA, Gilboa A (2019) A Causal role for the precuneus in network-wide theta and gamma oscillatory activity during complex memory retrieval. Elife 8:1-20. https://doi.org/ 10.7554/eLife.43114

Hebscher M, Ibrahim C, Gilboa A (2020) Precuneus stimulation alters the neural dynamics of autobiographical memory retrieval. Neuroimage 210:116575. https://doi.org/10.1016/j. neuroimage.2020.116575

Hopcroft JE, Tarjan R (1973) Efficient algorithms for graph manipulations. Communication of the ACM 16(6):372-378

Ishii R, Canuet L, Ishihara T, Aoki Y, Ikeda S, Hata M, Katsimichas T, Gunji A, Takahashi H, Nakahachi T, Iwase M, Takeda M (2014) Frontal midline theta rhythm and gamma power changes during focused attention on mental calculation: an meg beamformer analysis. Front Hum Neurosci 8:1-10. https://doi.org/ 10.3389/fnhum.2014.00406

Jacobs J (2014) Hippocampal theta oscillations are slower in humans than in rodents: implications for models of spatial navigation and memory. Philosophical Transactions of the Royal Society B: Biological Sciences. https://doi.org/10.1098/rstb.2013.0304

Jung TP, Makeig S, Humphries C, Lee TW, Mckeown MJ, Iragui V, Sejnowski TJ (2000) Removing electroencephalographic artifacts by blind source separation. Psychophysiology 37(2):163178. https://doi.org/10.1017/S0048577200980259

Kriegeskorte N, Kyle Simmons W, Bellgowan PS, Baker CI (2009) Circular analysis in systems neuroscience: the dangers of double dipping. Nat Neurosci 12(5):535-540. https://doi.org/10.1038/ nn. 2303

Kriegeskorte N, Lindquist MA, Nichols TE, Poldrack RA, Vul E (2010) Everything you never wanted to know about circular analysis, but were afraid to ask. J Cereb Blood Flow Metab 30(9):1551-1557. https://doi.org/10.1038/jcbfm.2010.86

Maris E, Oostenveld R (2007) Nonparametric statistical testing of EEG- and MEG-Data. J Neurosci Methods 164(1):177-190. https://doi.org/10.1016/j.jneumeth.2007.03.024 
McCormick C, Ciaramelli E, De Luca F, Maguire EA (2018) Comparing and contrasting the cognitive effects of hippocampal and ventromedial prefrontal cortex damage: a review of human lesion studies. Neuroscience 374:295-318. https://doi.org/10. 1016/j.neuroscience.2017.07.066

McCormick C, Barry DN, Jafarian A, Barnes GR, Maguire EA (2020) VmPFC Drives hippocampal processing during autobiographical memory recall regardless of remoteness. Cereb Cortex. https://doi.org/10.1093/cercor/bhaa172

Michel CM, Brunet D (2019) EEG source imaging: a practical review of the analysis steps. Front Neurol. https://doi.org/10. 3389/fneur.2019.00325

Miller J, Watrous AJ, Tsitsiklis M, Lee SA, Sheth SA, Schevon CA, Smith EH, Sperling MR, Sharan A, Asadi-Pooya AA, Worrell GA, Meisenhelter S, Inman CS, Davis KA, Lega B, Wanda PA, Das SR, Stein JM, Gorniak R, Jacobs J (2018) Lateralized hippocampal oscillations underlie distinct aspects of human spatial memory and navigation. Nat Commun. https://doi.org/10.1038/ s41467-018-04847-9

Nakazono T, Jun H, Blurton-Jones M, Green KN, Igarashi KM (2018) Gamma oscillations in the entorhinal-hippocampal circuit underlying memory and dementia. Neurosci Res 129:4046. https://doi.org/10.1016/j.neures.2018.02.002

Nyhus E, Curran T (2010) Functional role of gamma and theta oscillations in episodic memory. Neurosci Biobehav Rev 34(7):1023-1035. https://doi.org/10.1016/j.neubiorev.2009. 12.014

Oostenveld R, Fries P, Maris E, Schoffelen JM (2011) FieldTrip: open source software for advanced analysis of MEG, EEG, and invasive electrophysiological data. Comput Intell Neurosci. https://doi.org/ $10.1155 / 2011 / 156869$

Osipova D, Takashima A, Oostenveld R, Fernández G, Maris E, Jensen O (2006) Theta and Gamma Oscillations Predict Encoding and Retrieval of Declarative Memory. J Neurosci 26(28):7523-7531. https://doi.org/10.1523/JNEUROSCI.1948-06.2006

Özkurt TE, Schnitzler A (2011) A critical note on the definition of phase-amplitude cross-frequency coupling. J Neurosci Methods 201(2):438-443. https://doi.org/10.1016/j.jneumeth.2011.08.014

Pernet C, Garrido MI, Gramfort A, Maurits N, Michel CM, Pang E, Salmelin R, Schoffelen JM, Valdes-Sosa PA, Puce A (2020) Issues and Recommendations from the OHBM COBIDAS MEEG Committee for Reproducible EEG and MEG Research. Nat Neurosci. https://doi.org/10.1038/s41593-020-00709-0

Perrin F, Pernier J, Bertrand O, Echallier JF (1989) Spherical splines for scalp potential and current density mapping. Electroencephalogr Clin Neurophysiol 72(2):184-187. https://doi.org/10.1016/ 0013-4694(89)90180-6

Petersen SE, Posner MI (2012) The attention system of the human brain: 20 years after. Annu Rev Neurosci 35:73-89. https://doi. org/10.1146/annurev-neuro-062111-150525

Ptak R, Schnider A, Fellrath J (2017) The dorsal frontoparietal network: a core system for emulated action. Trends Cogn Sci 21(8):589-599. https://doi.org/10.1016/j.tics.2017.05.002

Ritchey M, Cooper RA (2020) Deconstructing the posterior medial episodic network. Trends Cogn Sci 24(6):451-465. https://doi. org/10.1016/j.tics.2020.03.006

Rolls ET, Joliot M, Tzourio-Mazoyer N (2015) Implementation of a new parcellation of the orbitofrontal cortex in the automated anatomical labeling atlas. Neuroimage 122:1-5. https://doi.org/10. 1016/j.neuroimage.2015.07.075

Rubega M, Carboni M, Seeber M, Pascucci D, Tourbier S, Toscano G, Van Mierlo P, Hagmann P, Plomp G, Vulliemoz S, Michel CM (2019) Estimating EEG source dipole orientation based on singular-value decomposition for connectivity analysis. Brain Topogr 32(4):704-719. https://doi.org/10.1007/s10548-018-0691-2

Sederberg PB, Kahana MJ, Howard MW, Donner EJ, Madsen JR (2003) Theta and gamma oscillations during encoding predict subsequent recall. J Neurosci 23(34):10809-10814. https://doi. org/10.1523/jneurosci.23-34-10809.2003

Sederberg PB, Schulze-Bonhage A, Madsen JR, Bromfield EB, McCarthy DC, Brandt A, Tully MS, Kahana MJ (2007) Hippocampal and neocortical gamma oscillations predict memory formation in humans. Cereb Cortex 17(5):1190-1196. https://doi.org/10.1093/ cercor/bhl030

Šidák Z (1967) Rectangular confidence regions for the means of multivariate normal distributions. J Am Stat Assoc 62(318):626-633. https://doi.org/10.1080/01621459.1967.10482935

Sirota A, Montgomery S, Fujisawa S, Isomura Y, Zugaro M, Buzsáki G (2008) Entrainment of neocortical neurons and gamma oscillations by the hippocampal theta rhythm. Neuron 60(4):683-697. https://doi.org/10.1016/j.neuron.2008.09.014

Stam CJ, van Straaten ECW (2012) Go with the Flow: Use of a Directed Phase Lag Index (DPLI) to Characterize patterns of phase relations in a large-scale model of brain dynamics. Neuroimage 62(3):1415-1428. https://doi.org/10.1016/j.neuroimage. 2012.05.050

Svoboda E, McKinnon MC, Levine B (2006) The functional neuroanatomy of autobiographical memory: a meta-analysis. Neuropsychologia 44(12):2189-2208. https://doi.org/10.1016/j.neuropsych ologia.2006.05.023

Tang W, Liu H, Douw L, Kramer MA, Eden UT, Hämäläinen MS, Stufflebeam SM (2017) Dynamic connectivity modulates local activity in the core regions of the default-mode network. Proc Natl Acad Sci USA 114(36):9713-9718. https://doi.org/10.1073/ pnas. 1702027114

Tort ABL, Kramer MA, Thorn C, Gibson DJ, Kubota Y, Graybiel AM, Kopell NJ (2008) Dynamic cross-frequency couplings of local field potential oscillations in rat striatum and hippocampus during performance of a t-maze task. Proc Natl Acad Sci USA 105(51):20517-20522. https://doi.org/10.1073/pnas.0810524105

Tzourio-Mazoyer N, Landeau B, Papathanassiou D, Crivello F, Etard O, Delcroix N, Mazoyer B, Joliot M (2002) Automated anatomical labeling of activations in SPM using a macroscopic anatomical parcellation of the mni mri single-subject brain. Neuroimage 15(1):273-289. https://doi.org/10.1006/nimg.2001.0978

Vann SD, Aggleton JP, Maguire EA (2009) What Does the Retrosplenial Cortex Do? Nat Rev Neurosci 10(11):792-802. https://doi. org/10.1038/nrn2733

Vogt BA (2016) Midcingulate cortex: structure, connections, homologies, functions and diseases. J Chem Neuroanat 74:28-46. https:// doi.org/10.1016/j.jchemneu.2016.01.010

Wang RWY, Chang WL, Chuang SW, Ning Liu I (2019) Posterior cingulate cortex can be a regulatory modulator of the default mode network in task-negative state. Sci Rep 9(1):1-12. https://doi.org/ 10.1038/s41598-019-43885-1

Yonelinas AP (2013) The hippocampus supports high-resolution binding in the service of perception, working memory and long-term memory. Behav Brain Res 254:34-44. https://doi.org/10.1016/j. bbr.2013.05.030

Zalesky A, Fornito A, Bullmore ET (2010) Network-based statistic: identifying differences in brain networks. Neuroimage 53(4):1197-1207. https://doi.org/10.1016/j.neuroimage. 2010. 06.041

Zeidman P, Maguire EA (2016) Anterior hippocampus: the anatomy of perception, imagination and episodic memory. Nat Rev Neurosci 17(3):173-182. https://doi.org/10.1038/nrn.2015.24

Publisher's Note Springer Nature remains neutral with regard to jurisdictional claims in published maps and institutional affiliations. 